\title{
Association between pulse pressure and acute kidney injury after transcatheter aortic valve implantation
}

Yoko Minakawa, MD; Motoko Kimura, MD; Motoki Namekawa, MD; Shuku Yamagata, MD; Shigeki Yamashita, MD Department of Anesthesiology, Kurashiki Central Hospital, Kurashiki, Japan

\section{【Background】}

Acute kidney injury (AKI), a complication after transcatheter aortic valve implantation (TAVI), has been found to be $\mathrm{KCH}$ independently associated with short-term and long-term mortality. Arterial stiffness is reported to be a strong predictor of cardiovascular events and renal dysfunction. As arterial stiffness increases, a pulse wave is transmitted and reflected more rapidly, resulting in higher pulse pressure (PP). PP can be therefore used as an index of arterial stiffness. The association between AKI after TAVI and PP remains unclear.

\section{【Aim】}

To identify the predictors of AKI after TAVI and clarify the association between PP and AKI after TAVI.

\section{【Methods】}

Study subjects: Patients who underwent TAVI for aortic stenosis under general anaesthesia

Study duration: $1^{\text {st }}$ Oct 2013 to $31^{\text {st }}$ Aug 2017

Exclusion criteria: Patients in whom perioperative peripheral PP (pPP) was not measured, those with atrial fibrillation, those with severe aortic regurgitation, and those requiring cardiopulmonary bypass as a rescue device or reoperation because of bleeding complications

Measurements: Perioperative pPP at rest, obtained using oscillometry over the brachial artery, as an index of arterial stiffness AKI was defined according to the Valve Academic Research Consortium (VARC)-2 criteria.

Statistical analysis: Univariate and multivariate logistic regression analyses for identifying the independent preoperative and intraoperative predictors of AKI.

\section{【Results】}

\begin{tabular}{|l|c|c|c|c|}
\hline & All (n=74) & AKI (n=33) & Non-AKI (n=41) & P-value \\
\hline STS score & $5.97[4.92-7.93]$ & $6.53[5.16-9.42]$ & $5.69[4.85-6.92]$ & 0.111 \\
\hline Age, years & $86[83-89]$ & $87[84-89]$ & $86[82-88]$ & 0.198 \\
\hline Male sex & $24(32.4)$ & $11(33.3)$ & $13(31.7)$ & 0.882 \\
\hline Height, cm & $148[143-156]$ & $146[143-157]$ & $148[144-154]$ & 0.636 \\
\hline Body weight, kg & $47.6[43.4-57.4]$ & $50.0[45.3-60.0]$ & $47.1[42.9-52.3]$ & 0.164 \\
\hline Baseline serum creatinine, $\mathrm{mg} / \mathrm{dL}$ & $0.96[0.74-1.09]$ & $0.97[0.77-1.13]$ & $0.93[0.73-1.07]$ & 0.427 \\
\hline eGFR, mL/min/1.73m ${ }^{2}$ & $50.9[37.6-62.9]$ & $50.3[34.2-60.9]$ & $51.2[38.8-64.8]$ & 0.350 \\
\hline NYHA classification III-IV & $17(24.3)$ & $10(30.3)$ & $7(17.9)$ & 0.271 \\
\hline BNP, pg/mL & $181[96-361]$ & $201[119-372]$ & $170[90-799]$ & 0.589 \\
\hline pPP, mmHg & $67[53-78]$ & $73[67-86]$ & $58[50-72]$ & $<0.001$ \\
\hline Comorbidities & & & & \\
\hline Diabetes mellitus & $21(28.4)$ & $11(33.3)$ & $10(24.4)$ & 0.396 \\
\hline Dyslipidemia & $42(56.8)$ & $21(63.6)$ & $21(51.2)$ & 0.284 \\
\hline Hypertension & $62(83.8)$ & $30(90.9)$ & $32(78.0)$ & 0.136 \\
\hline Old myocardial infraction & $15(20.3)$ & $7(21.2)$ & $8(19.5)$ & 0.857 \\
\hline Chronic kidney disease & $50(67.6)$ & $25(75.8)$ & $25(61.0)$ & 0.177 \\
\hline Congestive heart failure & $34(45.9)$ & $14(42.4)$ & $20(48.8)$ & 0.585 \\
\hline COPD & $8(10.8)$ & $3(9.1)$ & $5(12.2)$ & 0.669 \\
\hline Peripheral vascular disease & $20(27.0)$ & $11(33.3)$ & $9(22.0)$ & 0.303 \\
\hline Echocardiographic findings & & & & \\
\hline Ejection fraction, \% & $62[57.25-69]$ & $62[56-67]$ & $62[59-71]$ & 0.465 \\
\hline Mean aortic valve gradient, $\mathrm{mmHg}$ & $54[44-64]$ & $52[44-62]$ & $55[44-65]$ & 0.575 \\
\hline Aortic valve area, cm²/m ${ }^{2}$ & $0.47[0.38-0.53]$ & $0.47[0.38-0.53]$ & $0.47[0.39-0.53]$ & 0.974 \\
\hline Procedural parameters & & & & \\
\hline Transapical approach & $27(36.5)$ & $19(57.6)$ & $8(19.5)$ & $<0.001$ \\
\hline Contrast agent, mL & $89[65-111]$ & $80[63-113]$ & $90[71-110]$ & 0.556 \\
\hline RBC transfusion needed & $49(66.2)$ & $27(81.8)$ & $22(53.7)$ & 0.011 \\
\hline
\end{tabular}

STS: Society of Thoracic Surgeons, eGFR: estimated glomerular filtration rate, NYHA: New York Heart Association, COPD: chronic obstructive pulmonary disease

Continuous variables are reported as median $\left[25^{\text {th }}-75^{\text {th }}\right]$. Category variables are reported as number. (\%).

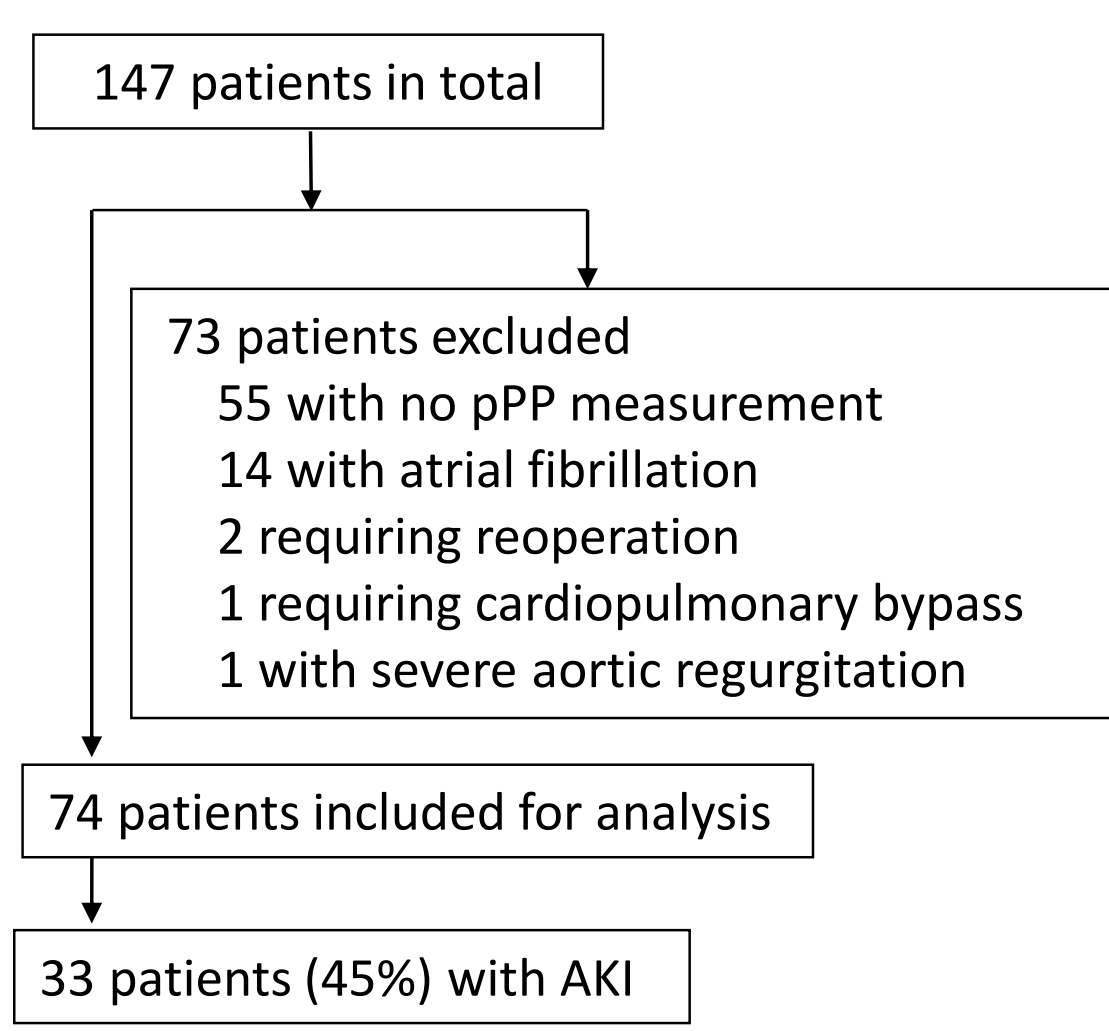

Fig. 1. Study flow diagram

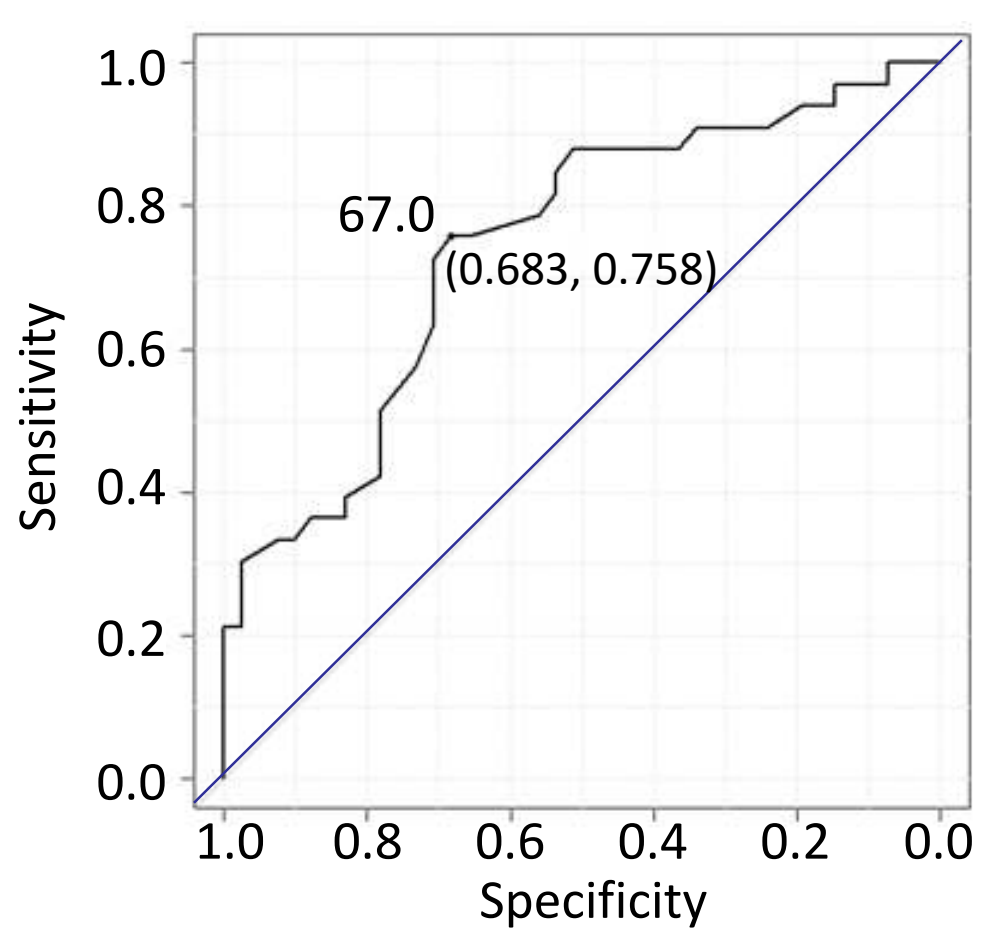

Fig. 2. $p P P R O C$ curve

Cut-off point of pPP: $67 \mathrm{mmHg}$ AUC: $0.743,95 \% \mathrm{Cl}[0.629-0.857]$ Sensitivity: 0.758 , Specificity: 0.683
Table 2 Risk factors for AKI development after TAVI

\begin{tabular}{lllll}
\hline \multirow{2}{*}{\multicolumn{1}{c}{ Variables }} & \multicolumn{2}{c}{ Univariate analysis } & \multicolumn{2}{c}{ Multivariate analysis } \\
\cline { 2 - 5 } & OR $(95 \% \mathrm{Cl})$ & $\mathrm{P}$-value & $\mathrm{OR}(95 \% \mathrm{Cl})$ & $\mathrm{P}$-value \\
\hline pPP, $\mathrm{mmHg}$ & $1.06(1.03-1.10)$ & $<0.001$ & $1.09(1.04-1.13)<0.001$ \\
Transapical approach & $5.60(1.99-15.8)$ & 0.001 & $13.1(3.34-50.9)$ & $<0.001$ \\
RBC transfusion needed & $3.89(1.32-11.4)$ & 0.014 & & \\
\hline
\end{tabular}

\section{【Conclusion】}

These results indicate that high pPP and the transapical approach can be predictors of AKI after TAVI. AKI was associated with longer length of stay in ICU and hospital after TAVI.
Table 3 Outcomes

\begin{tabular}{lcccc}
\hline & All $(\mathrm{n}=74)$ & AKI $(\mathrm{n}=33)$ & Non-AKI $(\mathrm{n}=41)$ & P-value \\
\hline ICU stay, hours & $50[27-74]$ & $62.5[50-100]$ & $27[24-51]$ & $<0.001$ \\
In-hospital mortality, $\mathrm{n}(\%)$ & $1(1.4)$ & $1(3)$ & $0(0)$ & 0.446 \\
Hospital stay after TAVI, days & $11[8-14]$ & $12[11-15]$ & $9[7-11]$ & $<0.001$ \\
6-month mortality, $\mathrm{n}(\%)$ & $2(2.7)$ & $2(6.1)$ & $0(0)$ & 0.212 \\
\hline
\end{tabular}
ICU: intensive care unit

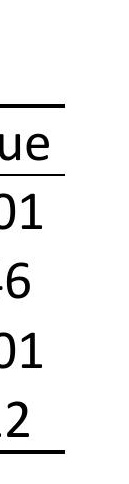

